\title{
Stereotactic body radiation therapy (SBRT) in patients with hepatocellular carcinoma and oligometastatic liver disease
}

\author{
Sabine Gerum¹, Christian Heinz ${ }^{1}$, Claus Belka¹, Franziska Walter', Philipp Paprottka², Enrico N. De Toni ${ }^{3}$ \\ and Falk Roeder ${ }^{1,4^{*}}$
}

\begin{abstract}
Background: To report our experience with SBRT in primary and secondary liver tumors.

Methods: We retrospectively analysed 55 patients (70 lesions) with a median follow-up of 10 months (range 1-57) treated from 2011 to 2016. All patients had not been eligible for other local treatment options. Median age was 64 years and 64\% were male. 27 patients (36 lesions) suffered from hepatocellular carcinoma (HCC, Child A:78\%, Child B:18\%, Child C:4\%), 28 patients (34 lesions) had oligometastatic liver disease (MD). Treatment planning was based on 4D-CT usually after placement of fiducials. Dose and fractionation varied depending on localization and size, most commonly $3 \times 12.5$ Gy (prescribed to the surrounding 65\%-isodose) in $56 \%$ and $5 \times 8 \mathrm{~Gy}$ (80\% isodose) in $20 \%$ of the treated lesions.
\end{abstract}

Results: Local recurrence was observed in 7 patients (13\%) and 8 lesions (11\%), resulting in estimated 1- and 2-year local control rates (LC) of 91 and 74\%. Estimated 1- and 2-year rates of Freedom from hepatic failure (FFHF) were 42 and 28\%. Number of lesions was predictive for LC and FFHF in the entire cohort. Estimated 1- and 2-year overall survival (OS) was 76 and $57 \%$. OS was significantly affected by number of treated lesions and performance status. In the HCC subgroup, pretreatment liver function and gender were also predictive for OS. Maximum acute non-hepatic toxicity was grade 1 in 16\% and grade 2 in 10\% of the patients. Three HCC patients (11\%) developed marked deterioration of liver function (grade 3/4).

Conclusions: SBRT resulted in high local control and acceptable survival rates in patients with HCC or MD not amendable to other locally-ablative treatment options with limited toxicity. Care should be taken in HCC patients with Child B cirrhosis.

Keywords: Liver, HCC, Oligometastatic, SBRT

\section{Background}

Primary and metastatic liver tumors are among the most common malignancies and tumour-related causes of death worldwide $[1,2]$. Treatment paradigms have changed dramatically in the last decades in favor of local treatments in primary liver cancers and oligometastatic (especially liver-confined) disease because of the evolving evidence for possible cure or at least long-term survival [3, 4].

\footnotetext{
* Correspondence: falk.roeder@med.uni-muenchen.de

'Department of Radiation Oncology, University Hospital LMU Munich,

Marchioninistr. 15, 81377 Munich, Germany

${ }^{4}$ CCU Molecular Radiation Oncology, German Cancer Research Center,

Heidelberg, Germany

Full list of author information is available at the end of the article
}

Treatment options range from liver transplantation (for $\mathrm{HCC}$ ) or extended surgical resections (for MD) to less invasive techniques like radiofrequency ablation (RFA), transarterial chemoembolization (TACE) or selective internal radiotherapy (SIRT). However, the potential benefit of such treatment options needs to be weighed against the possibility that local treatment results in impairment of liver function or liver failure, especially in the presence of an underlying liver disease, which is the background upon most primary malignancies arise [4]. Moreover, any of the mentioned treatments has its limitations. For example surgery is often limited by comorbidities or poor liver function [4] while lesions directly adjacent to major vessels or bile

(C) The Author(s). 2018 Open Access This article is distributed under the terms of the Creative Commons Attribution 4.0 International License (http://creativecommons.org/licenses/by/4.0/), which permits unrestricted use, distribution, and 
ducts are not well suited for RFA [5] and patients with portal vein thrombosis are not eligible for TACE [6].

Stereotactic body radiation therapy (SBRT) is a highly conformal technique of percutaneous radiation therapy delivered in a small number of large fractions [7]. It sufficiently spares dose to adjacent organs at risk due to its sharp dose fall-off outside the target, while adequate tumor control is maintained due to the enhanced biological effectivity of the large single doses. Stereotactic radiation approaches have been already successfully introduced into the treatment of primary and secondary brain and lung tumors and have shown to result in low toxicity and at least comparable outcome with regard to surgery [7-10]. Several groups have shown that SBRT can also be effectively employed with acceptable toxicity for the treatment of liver malignancies [11]. However, no randomized trials comparing SBRT to other local treatment options have been conducted so far, and only scarce prospective data on the employment of SBRT in the treatment of liver lesions are available. Moreover, no generally accepted criteria for patient selection or a generally accepted dose and fractionation concept exists. Therefore we report our experience with SBRT for primary and secondary liver tumors.

\section{Methods}

In our institution, SBRT has been used for the treatment of malignant liver lesions for the first time in 2011. Since then, an increasing number of patients have been treated each year (fig. 1). For the current analysis, we retrospectively selected and analyzed all patients affected by HCC or MD who underwent SBRT to 1-3 liver lesions. Indication for SBRT was seen in patients not eligible for other local treatment options according to multidisciplinary evaluation. Pretreatment investigations included MRI and/or contrast-enhanced biphasic liver CT, liver function tests for $\mathrm{HCC}$ patients and additional CT/ PET-CT staging for MD patients.

\section{Patient characteristics}

The entire cohort consisted of 55 patients with 70 treated lesions. Median age was 64 years (31-83), 64\% were male and the median Karnofsky performance score (KPS) was 90\% (60-100\%). 28 patients showed MD in whom 34 lesions were treated. Most of them suffered from colorectal cancer (32\%) followed by NSCLC (14\%) and soft-tissue sarcomas (14\%). 27 patients showed HCC in whom 36 lesions were treated. Underlying liver cirrhosis was present in all HCC patients, caused by viral hepatitis (B and C) in 10 patients (37\%), alcohol consumption in 5 (19\%), autoimmune hepatitis in $1(4 \%)$ and by unknown reason in 11 patients (41\%). Liver function was moderately restricted in the majority of them (Child A: 21[78\%], Child B: 5[18\%], Child C: 1[4\%]), Table 1. The patient with Child $\mathrm{C}$ cirrhosis presented with good performance score and was listed for liver

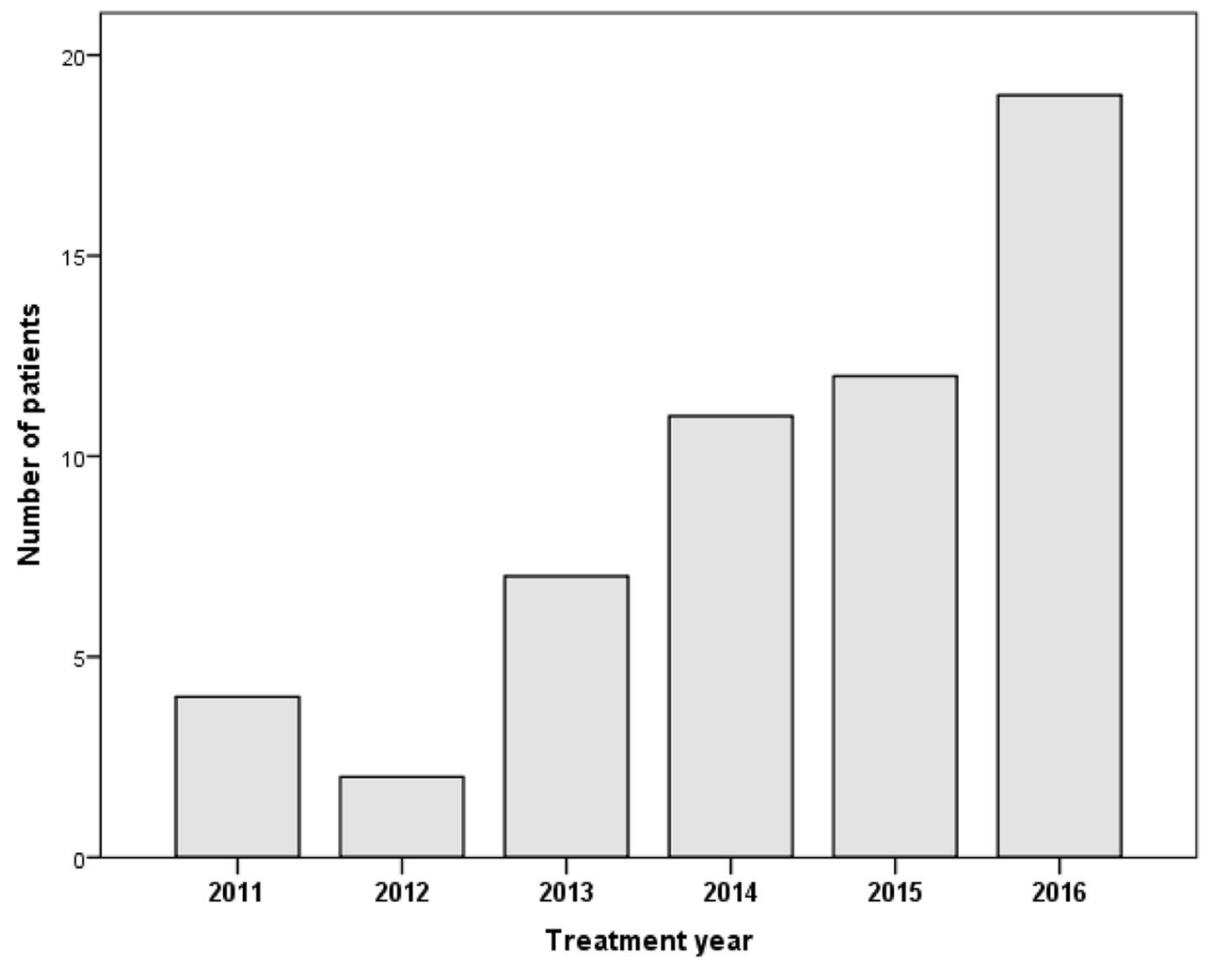

Fig. 1 Number of treatments per year 
Table 1 patient and treatment characteristics

\begin{tabular}{|c|c|c|c|}
\hline & entire cohort & $\mathrm{HCC}$ & $\mathrm{MD}$ \\
\hline number of patients & 55 & 27 & 28 \\
\hline number of lesions & 70 & 36 & 34 \\
\hline single/multiple lesions & $40 / 15$ & $18 / 9$ & $22 / 6$ \\
\hline gender (male/female) & $35 / 20$ & $19 / 8$ & $16 / 12$ \\
\hline age (median/range in years) & $64 / 31-83$ & $63 / 46-83$ & $67 / 31-80$ \\
\hline KPS (median/range in \%) & $90 / 60-100$ & $90 / 60-100$ & $95 / 70-100$ \\
\hline histology (CRC/LC/BC/S/O) & - & - & $10 / 4 / 4 / 2 / 9$ \\
\hline Child-Pugh stage (A/B/C) & - & $21 / 5 / 1$ & - \\
\hline fiducials (n/\%) & $42 / 76$ & $18 / 67$ & 24/86 \\
\hline abdominal compression ( $\mathrm{n} / \%)$ & $26 / 47$ & $14 / 52$ & $12 / 43$ \\
\hline GTV per patient (median/range in ccm) & 14.6/0.4-204 & $15.5 / 1.2-204$ & $13.25 / 0.4-98.2$ \\
\hline GTV per lesion (median/range in ccm) & $6.6 / 0.2-204$ & 7.5/0.9-204 & $6.2 / 0.2-98.2$ \\
\hline PTV per patient (median/range in ccm) & 75.5/17.7-511.6 & $92.7 / 17.7-511.6$ & $61.1 / 19.3-314$ \\
\hline PTV per lesion (median/range in $\mathrm{ccm}$ ) & $59 / 15-512$ & $61 / 15-512$ & $51 / 15-314$ \\
\hline dose concept $(3 \times 12.5 / 5 \times 8$ /other $)$ & 28/11/16 & $16 / 6 / 5$ & $12 / 5 / 9$ \\
\hline BED max (median/range) & 168.1/60.3-190 & $168.2 / 60.3-168.2$ & 118/80.4-190 \\
\hline actual treatment (SBRT/TACE+SBRT) & - & $12 / 15$ & - \\
\hline
\end{tabular}

HCC: hepatocellular carcinoma, MD: metastatic disease, KPS: Karnofsky performance score, CRC: colorectal cancer, LC: lung cancer, BC: breast cancer, S: sarcoma, o: other, GTV: gross tumor volume [cubic centimeters], PTV: planning target volume [cubic centimeters], BED: biologically equivalent dose, SBRT: stereotactic body radiotherapy, TACE: transarterial chemoembolization, all doses in [Gy]

transplantation, therefore our interdisciplinary tumorboard opted for SBRT as bridging.

\section{Treatment characteristics}

Treatment in HCC patients consisted of SBRT alone in 12 patients while 15 patients received selective TACE to the same lesions upfront to SBRT (within 6 weeks). Five patients received additional RFA treatments to different lesions prior to SBRT (within 6 weeks). 23/27 patients had a median of 2 (range 1-8) previous local treatments (surgery, RFA, TACE or SIRT). Treatment of metastatic patients consisted of SBRT alone in 25 patients or SBRT combined with surgery or RFA to different lesions (within 6 weeks) in 3 patients. Primary tumor was controlled in all patients with MD at the time of SBRT. Additive systemic therapy within three months from SBRT was given to 1 patient with HCC and 8 patients with MD.

\section{SBRT}

Prior to SBRT, 42 patients received CT-guided implantation of 1-3 fiducials (Visicoil ${ }^{\mathrm{TM}}$, IBA dosimetry or $\mathrm{MPB}^{\mathrm{s}}$, MPB Scherer Medizinprodukte) per lesion unless enhancement of lipiodol in patients with prior TACE $(n=8)$ or the presence of surgical clips $(n=5)$ were deemed sufficient to guide the procedure. Patients were immobilized using a vacuum pillow in combination with an alpha-cradle. Abdominal compression was used since 2014 ( $n=26,47 \%)$. Treatment planning was based on contrast-enhanced 4D-CT. Gross tumor volume (GTV) was contoured as the visible tumor on the free-breathing $\mathrm{CT}$ and on all respiratory phases of the 4D-CT supplemented by information from MRI if available. An internal target volume (ITV) was constructed and enlarged by an isotropic margin of $6 \mathrm{~mm}$ to obtain the planning target volume (PTV). Dose was prescribed to the PTV surrounding isodose in all patients. Prescription isodose, single dose and number of fractions depended on size and location of the lesions. Number of lesions did not influence prescription dose in general. The most common schemes were $3 \times 12.5$ Gy (65\%-isodose) in $56 \%$ and $5 \times 8$ Gy (80\%-isodose) in $20 \%$ of the treated lesions delivered every other day. Implanted fiducials or lipiodol enhancement were contoured accordingly to receive a fiducial or lipiodol ITV, which was used for daily patient set-up. Treatment was performed using daily CBCT image-guidance.

\section{Statistical and legal considerations}

Regular follow-up examinations (including physical examination, laboratory tests (liver function and tumor marker tests), MRI/CT of the liver) took place at our department or the departments of gastroenterology/oncology every three months for one year, every 6 months for the second and annually thereafter. Toxicity was scored retrospectively according to CTCAE v4.03. Because of the retrospective nature not all patients had received exactly the same laboratory tests. Therefore changes in 
Table 2 univariate analysis for LC, FFHF and OS

\begin{tabular}{|c|c|c|c|c|c|c|}
\hline & LC & & FFHF & & OS & \\
\hline & $\begin{array}{l}\text { 1-year } \\
\text { rate }\end{array}$ & $p$ value & $\begin{array}{l}1 \text {-year } \\
\text { rate }\end{array}$ & $p$ value & $\begin{array}{l}\text { 1-year } \\
\text { rate }\end{array}$ & $p$ value \\
\hline \multicolumn{7}{|l|}{ gender } \\
\hline male & $88 \%$ & 0.279 & $44 \%$ & 0.679 & $83 \%$ & 0.055 \\
\hline female & $94 \%$ & & $41 \%$ & & $67 \%$ & \\
\hline \multicolumn{7}{|l|}{ age } \\
\hline$\leq$ median (64 yrs) & $92 \%$ & 0.265 & $40 \%$ & 0.687 & $77 \%$ & 0.76 \\
\hline$>$ median & $90 \%$ & & $43 \%$ & & $75 \%$ & \\
\hline \multicolumn{7}{|l|}{ histology } \\
\hline $\mathrm{HCC}$ & $92 \%$ & 0.492 & $41 \%$ & 0.895 & $68 \%$ & 0.701 \\
\hline MD & $89 \%$ & & $40 \%$ & & $84 \%$ & \\
\hline \multicolumn{7}{|l|}{ KPS } \\
\hline$\leq 70$ & $100 \%$ & 0.425 & $42 \%$ & 0.579 & $50 \%$ & 0.028 \\
\hline$>70$ & $90 \%$ & & $42 \%$ & & $79 \%$ & \\
\hline \multicolumn{7}{|l|}{ GTV } \\
\hline$\leq$ median $(14.6 \mathrm{ccm})$ & $100 \%$ & 0.535 & $46 \%$ & 0.794 & $80 \%$ & 0.416 \\
\hline$>$ median & $88 \%$ & & $44 \%$ & & $77 \%$ & \\
\hline \multicolumn{7}{|l|}{ BED } \\
\hline$\leq 100$ & $87 \%$ & 0.651 & $38 \%$ & 0.419 & $75 \%$ & 0.806 \\
\hline$>100$ & $94 \%$ & & $48 \%$ & & $77 \%$ & \\
\hline \multicolumn{7}{|l|}{ lesions } \\
\hline single & $97 \%$ & 0.011 & $49 \%$ & 0.047 & $80 \%$ & $<0.001$ \\
\hline multiple & $67 \%$ & & $19 \%$ & & $40 \%$ & \\
\hline
\end{tabular}

LC: Local control, FFHF: Freedom from hepatic failure, OS: Overall survival, yrs.: years, HCC: hepatocellular carcinoma, MD: metastastic disease, KPS Karnofsky performance score, GTV: Gross tumor volume (measured per patient on free-breathing $(\mathrm{CT})$, ccm: cubic centimeters, BED: biological equivalent dose

hepatic laboratory tests without symptoms were not counted as toxicity. Marked deterioration of liver function was defined as change in CHILD-Pugh class from A to $\mathrm{B}$ or $\mathrm{B}$ to $\mathrm{C}$ in $\mathrm{HCC}$ patients or symptomatic changes in liver function in MD patients. Biological effective dose (BED) of the maximum PTV dose was calculated according to the LQ formalism: $B E D=n^{*} d^{*}(1+d /\{\alpha / \beta\})$ with $\mathrm{n}$ being the number of fractions, $\mathrm{d}$ the daily single fraction dose and alpha-beta for tumor tissue of $10 \mathrm{~Gy}$. Local control (LC) was defined as absence of tumor progression in the region of the treated lesion. Freedom from hepatic failure (FFHF) was defined as absence of tumor progression in the liver. All time-to-event data was calculated from the first day of SBRT using the Kaplan-Meier method. All endpoints and subgroup analyses are reported referring to patients (not lesions) if not otherwise specified. Differences in subgroups were assessed by the logrank test for univariate analysis. Due to the low number of events multivariate analysis was not performed. The pearsons test was used for evaluation of possible correlations between parameters. A $p$-value of $<0.05$ was defined as statistically significant. The analysis was in accordance to the declaration of Helsinki in its latest version and was approved by our independent Ethics committee.

\section{Results}

Median follow-up in all patients was 10 months (1-57) and 13 months in survivors. Since implementation of the technique at our center in 2011 we have seen a continuous increase in patient numbers per year resulting in $56 \%$ of the included patients treated in 2015/16 (fig. 1). Median GTV (measured on free-breathing CT) per patient was $14.6 \mathrm{ccm}(0.4-204)$ and $6.6 \mathrm{ccm}$ per lesion (0.2-204) and median PTV was $75.5 \mathrm{ccm}$ (17.7-511.6) per patient and $59 \mathrm{ccm}(15-512)$ per lesion.

LC

Local recurrence was observed in 7/55 patients (13\%) translating into estimated 1- and 2-year LC-rates of 91 and $74 \%$ (fig. 2). Median time to local failure in these 7 patients was 8 months (2-39). In univariate analysis, only the number of lesions was predictive for LC (1-year-LC single $97 \%$ vs. multiple $67 \%, p=0.011$, Fig. 2, Table 2). In the HCC group we observed estimated 1and 2-year LC-rates of $92 \%$. The number of lesions was the only factor with significant impact on LC (1-year-LC single $100 \%$ vs. multiple $71 \%, p=0.024)$. No significant difference was observed comparing patients with TACE + SBRT versus SBRT alone. In the MD group, we observed estimated 1- and 2-year LC-rates of 89 and 64\%. Improved LC was significantly associated with treatment of a single lesion (1-year-LC $95 \%$ vs. $0 \%, p=0.027$ ), BED $>150$ Gy (1-year-LC $100 \%$ vs. $82 \%, p=0.036$ ) and female gender (1-year-LC $100 \%$ vs. $78 \%, p=0.039)$. We further analyzed possible associations between the number of lesions and dose parameters (BED maximum, prescription dose) but did not find any significant correlations (data not shown).

If LC was analysed per lesion, we observed local failures in 8/70 lesions (11\%), translating into 1- and 2-year LC-rates of 91 and 74\%. No factors with significant impact on LC could be identified for the entire cohort or the HCC subgroup. However, regarding the MD subgroup, we observed a significant association of LC with GTV volume (1-year LC GTV $\leq 6.25 \mathrm{ccm} 100 \%$ vs. $80 \%$ with GTV > 6.25ccm; $p=0.041)$.

\section{FFHF}

$29 / 55$ patients (53\%) showed hepatic failure of whom only 1 had an isolated local failure, while 22 showed isolated failures outside the treated volume and 6 had combined failures. Estimated 1- and 2-year FFHF-rates were 42 and 28\% (fig. 3). Again, only the number of lesions (1-yr-FFHF single $49 \%$ vs. $19 \%$ multiple, $p=0.047$ ) was predictive for FFHF 

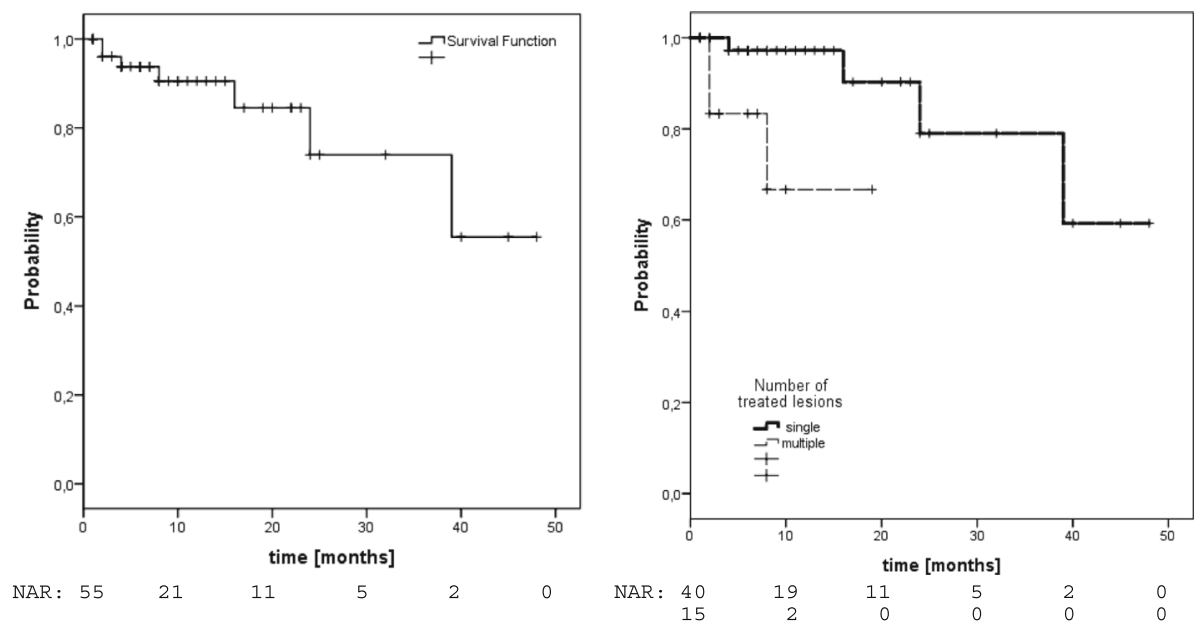

Fig. 2 Local control left: entire cohort, right: according to number of treated lesions

(Fig. 3, Table 2). In the HCC subgroup, we observed estimated 1- and 2-year FFHF-rates of $41 \%$. FFHF was significantly affected by the number of lesions (1-year-FFHF single $53 \%$ vs $15 \%$ in multiple, $p=0.01$ ). In the MD subgroup we found estimated 1- and 2-year FFHF-rates of 40 and $25 \%$. FFHF was significantly associated with performance status (1-year-FFHF KPS $\leq 700 \%$ vs. $43 \%$ with KPS > $70, p=0.006)$.

\section{OS}

16 patients $(29 \%)$ have died, translating into estimated 1- and 2-year OS-rates of 76 and 57\% (Fig. 4). Prognostic factors regarding OS were number of treated lesions (1-yr-OS single $88 \%$ vs $40 \%$ in multiple, $p<0.001$, Fig. 4 ) and performance status (1-yr-OS KPS $>7079 \%$ vs $50 \%$ KPS $\leq 70, p=0.028$, Fig. 4 ), see Table 2 . A trend was also observed for gender (1-yr-OS male $83 \%$ vs $67 \%$ female, $p=0.055)$. In the HCC subgroup we observed estimated
1- and 2-year OS-rates of 68 and 57\%. OS was significantly associated with number of treated lesions (1-year-OS single $84 \%$ vs $39 \%$ multiple,p $<0.001$ ), pretreatment liver function (1-year-OS Child-Pugh A 76\% vs. Child-Pugh B $28 \%, p=0.036)$ and gender (1-year-OS male $83 \%$ vs. female $43 \%, p=0.049$ ). In the MD subgroup we observed estimated 1- and 2-year OS-rates of 84 and $67 \%$. OS was significantly associated with performance status (1-year-OS KPS $>7090 \%$ vs. $33 \%$ KPS $\leq 70, p<$ $0.001)$ and number of treated lesions (1-year-OS single $90 \%$ vs. $42 \%$ multiple, $\mathrm{p}=0.036$ ).

\section{Toxicity}

CT-guided fiducial placement was feasible without any complications. SBRT treatment could be performed as planned in all except one patient (2\%) who prematurely finished radiation treatment due to humerus fracture. Maximum acute toxicity was grade 1 in 9 patients (16\%),
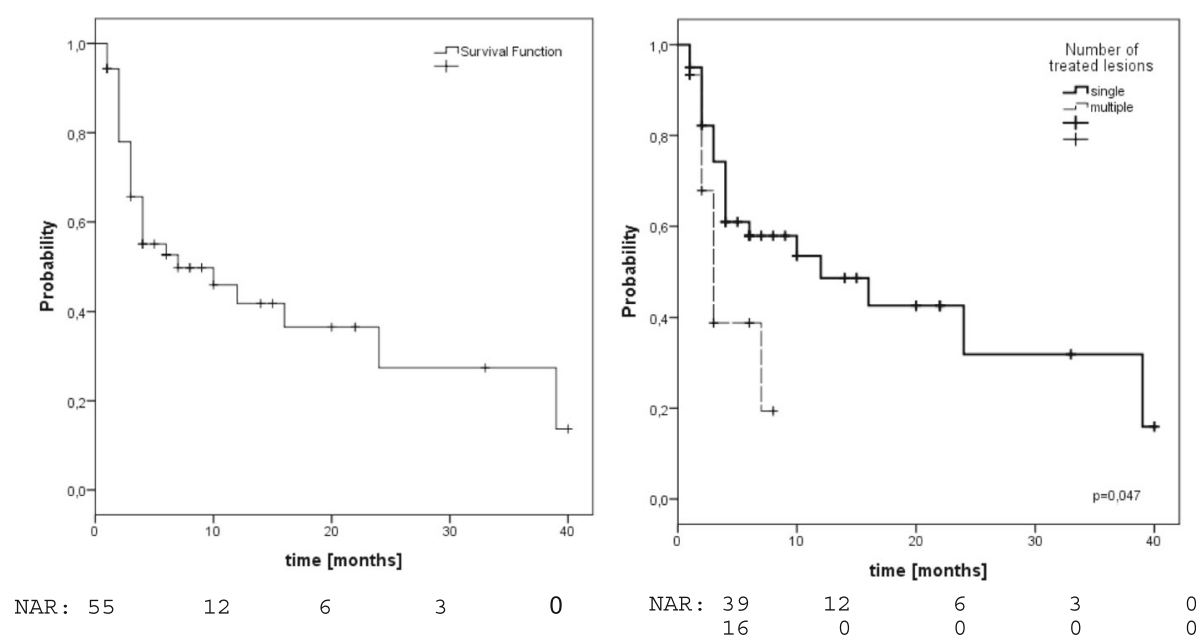

Fig. 3 Freedom from hepatic failure left: entire cohort, right: according to number of treated lesions 

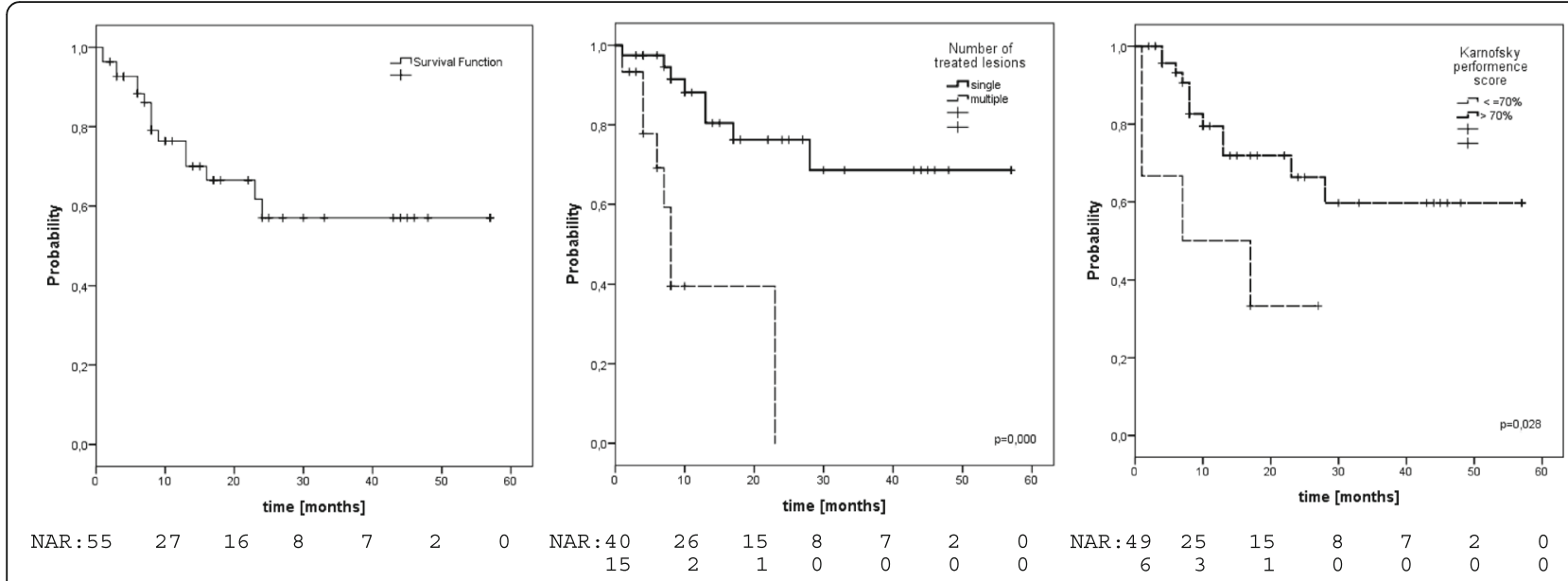

Fig. 4 Overall Survival left: entire cohort, middle: according to number of treated lesions, right: according to performance status

grade 2 in $6(10 \%)$, grade 3 in $2(4 \%)$ and grade 4 in 1 patient (2\%), see Table 3 . The most frequent side effects were fatigue and gastrointestinal symptoms like mild nausea or diarrhea. Three patients with HCC (11\%) developed marked deterioration of liver function without disease progression. Two had a decline in Child-Pugh stage from A to B. One patient with Child-Pugh C cirrhosis developed hepatic failure (grade 4) shortly after SBRT which was successfully treated by liver transplantation.

\section{Discussion}

\section{Outcome with SBRT}

Regarding LC, we observed 1- and 2-year-rates of 91 and $74 \%$ for the entire cohort. LC seemed slightly improved in the HCC group with 1- and 2-year-rates of $92 \%$ compared to the MD group with 1- and 2 -year-rates of 89 and 64\%, although this difference did not reach statistical significance. These results are in line with other series including both primary and secondary liver tumors treated with similar concepts. For example

Table 3 Acute toxicity

\begin{tabular}{|c|c|c|c|c|}
\hline & $\begin{array}{l}\text { CTCAE } \\
\text { ol }\end{array}$ & $\begin{array}{l}\mathrm{CTCAE}^{\circ} \\
\|\end{array}$ & $\begin{array}{l}\mathrm{CTCAE}^{\circ} \\
\text { III }\end{array}$ & $\begin{array}{l}\text { CTCAE }^{\circ} \\
\text { IV }\end{array}$ \\
\hline & \multicolumn{4}{|l|}{ n (\%) } \\
\hline nausea (n/\%) & $4(7)$ & $4(7)$ & & \\
\hline fatigue & $3(5)$ & & & \\
\hline pneumonitis & $1(2)$ & & & \\
\hline tachyarrhythmia & $1(2)$ & & & \\
\hline cholangitis & & $1(2)$ & & \\
\hline diarrhea & & $1(2)$ & & \\
\hline $\begin{array}{l}\text { severe deterioration of liver } \\
\text { function }\end{array}$ & n.a. & n.a. & $2(4)$ & $1(2)$ \\
\hline
\end{tabular}

n.a.: not applicable, CTCAE: common toxicity criteria for adverse events Version 4.03
Mendez-Romero et al. [12] described their results of a phase I-II trial including 25 patients with HCC or MD treated mainly with a $3 \times 12.5 \mathrm{~Gy}$ concept and found 1and 2-year LC-rates of 91 and $82 \%$. If HCC and MD are considered separately, our results are still in the published range of 75-100\% 1-year-LC in HCC [4] and 7194\% 1-year-LC in MD patients [13] reported in recent major series (Table 4).

With regard to OS, we observed 1- and 2-year-rates of 76 and $57 \%$ for the entire cohort. OS seemed to be slightly better in the MD group with 1- and 2-year OS-rates of 84 and $67 \%$ compared to the HCC group with 1 - and 2-year OS-rates of 68 and 57\%, although this difference did not reach statistical significance. Mendez-Romero et al. [12] described similar outcomes with 1- and 2-year OS-rates of 82 and 54\% for the whole group, 85 and $62 \%$ for patients with MD and 75 and $40 \%$ for patients with HCC. Moreover, our results are in the range of published results focusing on primary or secondary liver tumors with 1- and 2-year OS-rates of $62-85 \%$ and $38-70 \%$ in metastatic patients [3] and 55100 and $53 \%-69 \%$ in HCC patients [4].

In contrast to the encouraging rates for LC and OS, we observed rather poor 1- and 2-year FFHF-rates of 42 and $28 \%$ for the entire cohort, which were mainly driven by intrahepatic outfield failures indicating a high risk for the development of new lesions in these heavily pretreated patients. This pattern was seen in patients with HCC (1- and 2-year FFHF 42\%) as well as in patients with MD (1- and 2-year FFHF 40 and 25\%) although more pronounced in the latter group. Similar results have been reported by others for both entities. For example Yoon et al. [14] found a crude rate of $63 \%$ intrahepatic outfield failures and $71 \%$ hepatic failures in total, translating into a 1-year hepatic-failure-free-survival-rate of $52 \%$ in their cohort of 92 patients with HCC treated with SBRT. Chang et al. [15] reported a crude rate of 
Table 4 Selected SBRT series in patients with primary and secondary liver tumors

\begin{tabular}{|c|c|c|c|c|c|c|}
\hline $\begin{array}{l}\text { Author, Year } \\
\text { Study-Type }\end{array}$ & $\begin{array}{l}\mathrm{n} \text { (patients) } \\
\mathrm{n} \text { (lesions) }\end{array}$ & $\begin{array}{l}\mathrm{n} \text { (primary LT) } \\
\mathrm{n} \text { (metastases) }\end{array}$ & $\begin{array}{l}\text { GTV volume } \\
\text { (median/range) }^{\mathrm{a}}\end{array}$ & $\begin{array}{l}1 \mathrm{yr}-\mathrm{LC}(\%) \\
\text { (HCC/MD) }\end{array}$ & $\begin{array}{l}1 \text { yr-OS (\%) } \\
\text { (HCC/MD) }\end{array}$ & $\begin{array}{l}\text { Toxicity } \\
\text { Gr3+ (\%) }\end{array}$ \\
\hline $\begin{array}{l}\text { Mendez-Romero et al. } 2006 \\
\text { Phase I/II }\end{array}$ & $\begin{array}{l}25 \\
45\end{array}$ & $\begin{array}{l}\text { HCC: } 11 \\
\text { MD: } 34\end{array}$ & $3.5(0.5-7.2)$ & $\begin{array}{l}94 \\
(75 / 100)\end{array}$ & $\begin{array}{l}82 \\
(75 / 85)\end{array}$ & 12.5 \\
\hline $\begin{array}{l}\text { Goodman et al. } \\
2010 \\
\text { Phase I }\end{array}$ & $\begin{array}{l}26 \\
40\end{array}$ & $\begin{array}{l}\text { HCC/CCC: } 7 \\
\text { MD: } 19\end{array}$ & $32.6(0.8-146.6)$ & 77 & 62 & 0 \\
\hline Own data & $\begin{array}{l}55 \\
70\end{array}$ & $\begin{array}{l}\text { HCC: } 27 \\
\text { MD: } 28\end{array}$ & $6.6(0.2-204)$ & $\begin{array}{l}91 \\
(92 / 89)\end{array}$ & $\begin{array}{l}76 \\
(68 / 84)\end{array}$ & $5^{\mathrm{b}}$ \\
\hline $\begin{array}{l}\text { Bujold et al. } \\
2013 \\
\text { Phase I/II }\end{array}$ & 102 & HCC only & $7.2(1.4-23.1)$ & 87 & 55 & 30 \\
\hline $\begin{array}{l}\text { Lasley et al. } \\
2015 \\
\text { Phase I/II }\end{array}$ & $\begin{array}{l}59 \\
59\end{array}$ & HCC only & $33.6(2.2-107.3)$ & $\begin{array}{l}91 \text { (Child A) } \\
82 \text { (Child B) }\end{array}$ & $\begin{array}{l}94 \text { (Child A) } \\
57 \text { (Child B) }\end{array}$ & $\begin{array}{l}11 \text { (Child A) } \\
38 \text { (Child B) }\end{array}$ \\
\hline Own data & $\begin{array}{l}27 \\
36\end{array}$ & HCC only & $7.5(0.9-204)$ & 92 & 68 & $11^{\mathrm{b}}$ \\
\hline $\begin{array}{l}\text { Scorsetti et al. } \\
2015 \\
\text { Phase I/II }\end{array}$ & $\begin{array}{l}42 \\
52\end{array}$ & MD only & $3.5(1.1-5.4)$ & 91 (2 yr) & 65 (2 yr) & 0 \\
\hline $\begin{array}{l}\text { Andratschke et al. } \\
2015 \\
\text { Retro, pooled }\end{array}$ & $\begin{array}{l}74 \\
91\end{array}$ & MD only & n.r. & 75 & 77 & 0 \\
\hline Own data & $\begin{array}{l}28 \\
34\end{array}$ & MD only & $6.2(0.2-98.2)$ & 89 & 84 & 0 \\
\hline
\end{tabular}

n: number, LT: liver tumors, GTV: gross tumor volume per lesion, yr.: year, LC: local control, OS: overall survival, HCC: hepatocellular carcinoma, MD: metastatic disease, Gr3+: grade $3+{ }^{a}$ in cubic centimeters, ${ }^{\text {b}}$ : deterioration of liver function, no other grade 3+ toxicity, n.r.: not reported

$68 \%$ intrahepatic outfield failures in their study on 65 patients with colorectal liver metastases.

\section{Prognostic factors}

We analyzed our entire cohort and both subgroups (HCC and MD) with regard to possible prognostic factors for each endpoint. Regarding LC, lesion size [16$18]$ and dose of SBRT [5, 15, 16, 18-20] have been the most consistently reported prognostic factors for both groups. Although a variety of definitions and tresholds have been used and some studies did not found any associations at all [19-22]. We could not confirm a statistically significant association of GTV volume or BED with $\mathrm{LC}$ regarding the entire cohort and the subgroup of HCC patients. However, we observed a significantly reduced LC-rate with lower BED and larger GTV volume in the subgroup of patients with MD, thus emphasizing the findings of others suggesting a relationship between LC and lesion size and/or dose at least in metastatic patients. Interestingly, the number of lesions was the main factor in our study with a significant impact on LC, however this might be simply due to statistical reasons as patients with more lesions obviously have a high probability to fail at least in one.

Regarding OS, an even larger variety of factors with possible impact have been described in the literature, including gender [23], number of lesions [22], lesion diameter [15, 23], GTV volume [16, 17] and dose [24] for $\mathrm{HCC}$ and/or MD as well as Child-Pugh stage for HCC $[17,24]$ and histology for MD [16, 22]. However, the prognostic value of those factors is far from being consistently evident as negative or even opposing results have been reported also for any of the mentioned factors $[15,16,20,21,23,25]$. In our study, we identified the number of lesions and performance status as factors associated with OS for the entire cohort. In the HCC subgroup Child-Pugh stage and gender were additional factors with significant impact. Although conflicting data exists, it seems reasonable to assume that patients with multiple lesions are at higher risk for the development of consecutive lesions with consequently reduced survival, especially in a patient group like ours with limited salvage options. This is further supported by our data with regard to the clearly reduced FFHF in patients with multiple lesions. The same assumption seems true for performance status which has been shown in many other oncological situations to be a key factor in predicting outcome [26, 27]. Regarding the HCC subgroup, it has been shown that OS is clearly associated with Child-Pugh stage [28]. It therefore seems no surprise that OS of patients with HCC lesions (which developed on the basis of advanced liver cirrhosis) is affected by the severity of the underlying cirrhosis. In contrast, the observed negative influence of female gender on OS of 
HCC patients is difficult to explain. Huertas et al. [23] reported a similar result in their HCC series even according to multivariate analysis, however Yamashita et al. [25] found the opposite association leaving this question unanswered.

\section{Toxicity}

Given the high rate of outfield failures with the need for salvage treatments, toxicity and preservation of liver function are of important value in the decision process for locally-ablative treatments. With our approach, we observed high treatment compliance with predominantly mild toxicities mainly including fatigue and nausea. Three patients with HCC (11\%) developed marked deterioration of liver function. Similar results have been published in most other series. For patients treated for $\mathrm{MD}$, acute and late grade3+ toxicities are reported in the range of $0-16 \%$ and $0-5 \%$ [3]. Similar to our results, Andratschke et al. [16] reported mild side effects mainly consisting of fatigue and nausea but no grade3+ reactions in their series of 74 patients treated with comparable dose and fractionation concepts. In patients with HCC higher complications rates have been observed. Acute grade3+ toxicities ranged from 5 to $37 \%$ including up to $7 \%$ deaths [4], mainly in Child-Pugh B patients. Decline in Child-Pugh class has also been reported in $13-29 \%[12,20,24]$, although some authors described a marked recovery over time [20]. Our toxicity rate seem to compare favorably with those rates, however some of these studies included larger lesions resulting in more dose to normal liver tissue and a higher percentage of Child-Pugh B patients. Both factors have been shown to be associated with toxicity $[24,29]$. For example Andolino et al. [24] described progressive liver dysfunction in $4 / 8$ patients with Child-Pugh $\geq 8$ of whom 2 could be salvaged by transplant but 2 died. They concluded to further treat Child-Pugh B patients only if listed for transplantation. Lasley et al. [29] observed grade 3/4 liver toxicity of $11 \%$ in patients with Child-Pugh A compared to $38 \%$ in Child-Pugh B patients and further excluded patients with Child-Pugh $\geq 8$ from treatment. Finally, Culleton et al. [30] analyzed specifically patients with Child-Pugh $\mathrm{B} / \mathrm{C}$ and described a decline of $\geq 2$ points in $63 \%$ at 3 months. Therefore SBRT treatment should be used with caution or restricted in dose in patients with already restricted pretreatment liver function (Child Pugh B) while SBRT seems to be generally well tolerated in patients with Child Pugh A or in patients with MD. Moreover, a recent systematic review including 5 studies with 392 patients suffering from primary and secondary liver tumors treated with SBRT demonstrated well-preserved post-treatment quality-of-life at least comparable or even favorable compared to other surgical or non-surgical approaches [1].

\section{Limitations}

Clearly our analysis has some limitations, namely its retrospective nature, the small sample size, the mixed cohort and the rather short follow-up. However, in the absence of prospective randomized trials and only a limited number of prospective studies reported in the literature our experience may help clinicians and researchers to guide their further decisions.

\section{Conclusions}

SBRT resulted in high LC and acceptable survival rates in patients with HCC or MD not amendable to other locally-ablative treatments. However, especially patients with multiple lesions are at high risk for intrahepatic outfield failures indicating a possible need for additional therapies. OS was predicted by number of lesions and performance status as well as pretreatment liver function in HCC patients. Toxicity was generally mild. High grade toxicity was restricted to patients with HCC suffering from underlying cirrhosis Child-Pugh class B indicating the need for special attention in those patients.

\section{Abbreviations \\ 4D-CT: Four-dimensional computed tomography; BED: Biologically effective dose; CBCT: Cone beam computed tomography; ccm: Cubic centimeters; CT: Computed tomography; CTCAE: Common toxicity criteria for adverse events; FFHF: Freedom from hepatic failure; GTV: Gross tumor volume; Gy: Gray; HCC: Hepatocellular carcinoma; ITV: Internal target volume; KPS: Karnofsky performance score; LC: local control; MD: Oligometastatic disease; MRI: Magnetic resonance imaging; OS: Overall survival; PET- CT: Positron-emission-tomography with computed tomography; PTV: Planning target volume; RFA: Radiofrequency ablation; RILD: Radiation induced liver disease; SBRT: Stereotactic body radiation therapy; SIRT: Selective internal radiotherapy; TACE: Transarterial chemoembolization}

Funding

The study did not receive any funding.

Availability of data and materials

The datasets used and/or analysed during the current study are available from the corresponding author on reasonable request.

\section{Authors contributions}

SG performed data acquisition and participated in patient treatment, statistical analysis and in drafting the manuscript. $\mathrm{CH}$ participated in treatment of the patients and statistical analysis. FW, PP, ED treated the patients and participated in data acquisition. CB revised the manuscript critically. FR participated in data acquisition, statistical analysis, treatment of the patients, drafting the manuscript and critically reviewed the data and the manuscript. All authors read and approved the final manuscript

\section{Ethics approval and consent to participate}

The study was approved by the Ethics committee of the University of Munich (LMU), reference number 617-16.

Competing interests

The authors declare that they have no competing interests.

\section{Publisher's Note}

Springer Nature remains neutral with regard to jurisdictional claims in published maps and institutional affiliations. 


\section{Author details}

Department of Radiation Oncology, University Hospital LMU Munich, Marchioninistr. 15, 81377 Munich, Germany. ${ }^{2}$ Department of Radiology, University Hospital LMU Munich, Marchioninistr. 15, 81377 Munich, Germany. ${ }^{3}$ Department of Internal Medicine, University Hospital LMU Munich, Marchioninistr. 15, 81377 Munich, Germany. ${ }^{4}$ CCU Molecular Radiation Oncology, German Cancer Research Center, Heidelberg, Germany.

Received: 28 February 2018 Accepted: 16 May 2018

Published online: 29 May 2018

\section{References}

1. Mutsaers A, Greenspoon J, Walker-Dilks C, Swaminath A. Systematic review of patients reported quality of life following stereotactic ablative radiotherapy for primary and metastatic liver cancer. Radiat Oncol. 2017;12:110

2. Bosch FX, Ribes J, Diaz M, Cleries R. Primary liver cancer: worldwide incidence and trends. Gastroenterology. 2004;127:5-16.

3. Goodman KA, Kavanagh BD. Stereotactic body radiotherapy for liver metastases. Sem Radiat Oncol. 2017:27:240-6.

4. Murray LJ, Dawson LA. Advances in stereotactic body radiation therapy for hepatocellular carcinoma. Sem Radiat Oncol. 2017;27:247-55.

5. Kalogeridi MA, Zygogianni A, Kyrgias G, Kouvaris J, Chatziioannou S, Kelekis $\mathrm{N}$, Kouloulias $\mathrm{V}$. Role of radiotherapy in the management of hepatocellular carcinoma: a systematic review. World J Hepatol. 2015;7:101-12.

6. Waller LP, Deshpande V, Pyrsopoulos N. Hepatocellular carcinoma: a comprehensive review. World J Hepatol. 2015;7:2648-63.

7. Guckenberger M, Andratschke N, Alheit H, Holy R, Moustakis C, Nestle U, Sauer O. Definition of stereotactic body radiotherapy: principles and practice for the treatment of stage I non-small cell lung cancer. Strahlenther Onkol. 2014;190:26-33.

8. El-Khatib M, El Majdoub F, Hunsche S, Hoevels M, Kocher M, Sturm V, Maarouf M. Stereotactic LINAC radiosurgery for the treatment of typical intracranial meningiomas. Efficacy and safety after a follow-up of over 12 years. Strahlenther Onkol. 2015;191:921-7.

9. Kocher M, Wittig A, Piroth MD, Treuer H, Seegenschmiedt H, Ruge M, Grosu AL, Guckenberger M. Stereotactic radiosurgery for treatment of brain metastases. A report of the DEGRO working group on stereotactic radiotherapy. Strahlenther Onkol. 2014;190:521-32.

10. Tanadini-Lang S, Rieber J, Filippi AR, et al. Nomogram based overall survival prediction in stereotactic body radiotherapy for oligo-metastatic lung disease. Radiother Oncol. 2017;123:182-8.

11. Herfarth KK, Debus J, Lohr F, Bahner ML, Rhein B, Fritz P, Höss A, Schlegel W, Wannenmacher MF. Stereotactic single-dose radiation therapy of liver tumors: results of a phase I/II trial. J Clin Oncol. 2001;19:164-70.

12. Mendez Romero A, Wunderink W. Hussain et al. stereotactic body radiation therapy for primary and metastatic liver tumors: a single institution phase III study. Acta Oncol. 2006:45:831-7.

13. Comito T, Clerici E, Tozzi A, D'Agostino G. Liver metastases and SBRT: a new paradigm ? Rep Pract Oncol Radiother. 2015:20:464-71.

14. Yoon SM, Lim YS, Park MJ, et al. Stereotactic body radiation therapy as an alternative treatment for small hepatocellular carcinoma. PLoS One. 2013;8:e79854

15. Chang DT, Swaminath A, Kozak M, et al. Stereotactic body radiotherapy for colorectal liver metastases - a pooled analysis. Cancer. 2011;117:4060-9.

16. Andratschke NH, Nieder C, Heppt F, Molls M, Zimmermann F. Stereotactic radiation therapy for liver metastases: factors affecting local control and survival. Radiat Oncol. 2015;10:69.

17. Kwon JH, Bae SH, Kim JY, et al. Long-term effect of stereotactic body radiation therapy for primary hepatocellular carcinoma ineligible for local ablation therapy or surgical resection. BMC Cancer. 2010;10:475.

18. Scorsetti M, Comito T, Cozzi L, et al. The challenge of inoperable hepatocellular carcinoma (HCC): results of a single-institutional experience on stereotactic body radiation therapy (SBRT). J Cancer Res Clin Oncol. 2015; 141:1301-9.

19. Wahl DR, Stenmark MH, Tao Y, et al. Outcomes after stereotactic body radiotherapy or radiofrequency ablation for hepatocellular carcinoma. J Clin Oncol. 2016:34:452-9.

20. Bujold A, Massey CA, Kim JJ, et al. Sequential phase I and II trials of stereotactic body radiotherapy for locally advanced hepatocellular carcinoma. J Clin Oncol. 2013;31:1631-9.
21. Scorsetti M, Comito T, Tozzi A, et al. Final results of a phase II trial for stereotactic body radiation therapy for patients with inoperable liver metastases from colorectal cancer. J Cancer Res Clin Oncol. 2015;141: $543-53$.

22. Habermehl D, Herfarth KK, Bermejo JL, et al. Single-dose radiosurgical treatment for hepatic metastases - therapeutic outcome of 138 treated lesions from a single institution. Radiat Oncol. 2013;8:175.

23. Huertas A, Baumann AS, Saunier-Kubs F, et al. Stereotactic body radiation therapy as an ablative treatment for inoperable hepatocellular carcinoma. Radiother Oncol. 2015;115:211-6.

24. Andolino DL, Johnson CS, Maluccio M, et al. Stereotactic body radiotherapy for primary hepatocellular carcinoma. Int J Radiat Oncol Biol Phys. 2011;81: e447-53.

25. Yamashita H, Onishi $H$, Murakami N, et al. Survival outcomes after stereotactic body radiotherapy for 79 japanese patients with hepatocellular carcinoma. J Radiat Res. 2015;56:561-7.

26. Lancia A, Ingross G, Carosi A, et al. Oligometastatic cancer: stereotactic ablative radiotherapy for patients affected by isolated body metastases. Acta Oncol. 2017;56:1621-5.

27. Fode MM, Hoyer M. Survival and prognostic factors in 321 patients treated with stereotactic body radiotherapy for olio-metastases. Radiother Oncol. 2015:114:155-60.

28. D'Amico G, Garcia-Tsao G, Pagliaro L. Natural history and prognostic indicators of survival in cirrhosis: a systematic review of 118 studies. J Hepatol. 2006;44:217-31.

29. Lasley FD, Maninna EM, Johnson CS, et al. Treatment variables related to liver toxicity in patients with hepatocellular carcinoma, Child-Pugh class a and $B$ enrolled in a phase 1-2 trial of stereotactic body radiation therapy. Pract Radiat Oncol. 2015;5:e443-9.

30. Culleton $\mathrm{S}$, Jiang $\mathrm{H}$, Haddad $\mathrm{CR}$, et al. Outcomes following definitive stereotactic body radiotherapy for patients with Child-Pugh B or C hepatocellular carcinoma. Radiother Oncol. 2014;111:412-7.

\section{Ready to submit your research? Choose BMC and benefit from:}

- fast, convenient online submission

- thorough peer review by experienced researchers in your field

- rapid publication on acceptance

- support for research data, including large and complex data types

- gold Open Access which fosters wider collaboration and increased citations

- maximum visibility for your research: over $100 \mathrm{M}$ website views per year

At BMC, research is always in progress.

Learn more biomedcentral.com/submissions 